\title{
A CHARACTERIZATION OF PREORDERED FIELDS WITH THE WEAK HILBERT PROPERTY
}

\author{
ZENG GUANGXIN
}

(Communicated by Louis J. Ratliff, Jr.)

\begin{abstract}
By introducing a new notion "local density", we show the following result: Let $(K, S)$ be a preordered field, then $(K, S)$ has the weak Hilbert property if and only if $(K, S)$ is locally dense. From this, a theorem of McKenna in reference [1] and one of Prestel in reference [2] will be easily proved.
\end{abstract}

1. Introduction. In reference [1], McKenna introduced the concept of ordered fields with the weak Hilbert property to mean the class of ordered fields for which Hilbert's 17th problem is affirmative. In order to characterize ordered fields with the weak Hilbert property, McKenna has showed the following important result:

PROPOSITION 1. If $K$ is an ordered field with real closure $\bar{K}$, then $K$ has the weak Hilbert property if and only if $K$ is dense in $\bar{K}$ (cf. Theorems 2 and 3 in [1]).

Afterwards, in 1979, Alexander Prestel discussed formally real fields with the weak Hilbert property, and has obtained the following further result [2]:

PROPOSITION 2. If $K$ is a formally real field which has only a finite number of orderings, then $K$ has the weak Hilbert property if and only if $K$ is dense in every real closure of $K$.

However, the "only if" part of Proposition 2 will not hold if the hypothesis "has only a finite number of orderings" is omitted. Indeed, we may show that the formally real field $\mathbf{R}(t)$, where $t$ is transcendental over the field $\mathbf{R}$ of real numbers, has the weak Hilbert property, but it is not dense in any of its real closures [8]. Thereby, in general, "density" is a stronger concept than "the weak Hilbert property". The question naturally arises: What is a new notion that is similar to density and is exactly equivalent to the weak Hilbert property? In this paper, we introduce a new notion, the so-called local density, and show the equivalence between this notion and the weak Hilbert property.

For generality, in this paper we study preordered fields, because both ordered fields and formally real fields are special and extreme examples of preordered fields. A preordered field is a pair $(K, S)$ where $K$ is a field and $S$ is a subset of $K$ satisfying $-1 \notin S, S+S \subseteq S, S \cdot S \subseteq S, K^{2} \subseteq S$. In this case we call $S$ a preordering of $K$. Hence every preordering of $K$ contains the set $\sum K^{2}$ of sums of squares in $K$.

Received by the editors January 27, 1987 and, in revised form, April 23, 1987.

1980 Mathematics Subject Classification (1985 Revision). Primary 12D15, 12J15.

Key words and phrases. Preordered fields, the weak Hilbert property, definite rational functions, local density. 
An ordering $P$ of $K$ is called an ordering of $(K, S)$, if $S \subseteq P$. Let $X_{K}(S)$ be the set of orderings of $(K, S)$, then $X_{K}(S) \neq \varnothing$. With the so-called Harrison topology, the topological space $X_{K}(S)$ is compact, Hausdorff and totally disconnected, and its basis for the topology is given by open sets $H\left(a_{1}, \ldots, a_{n}\right)=\{P \in$ $\left.X_{K}(S) \mid a_{1}, \ldots, a_{n} \in P\right\}$.

An ordered field $\left(K^{*}, P^{*}\right)$ is said to be an ordered extension of $(K, S)$, if $K \subseteq K^{*}$ and $S \subseteq P^{*}$. A real closed field $(\bar{K}, \bar{P})$ is said to be a real closure of $(K, S)$, if $\bar{K}$ is an algebraic extension of $K$ and $S \subseteq \bar{P}$.

Let $K\left(X_{1}, \ldots, X_{m}\right)$ be the rational function field over $K$ in $m$ variables $X_{1}, \ldots, X_{m}$ and $f\left(X_{1}, \ldots, X_{m}\right) \in K\left(X_{1}, \ldots, X_{m}\right)$. We say $f\left(X_{1}, \ldots, X_{m}\right)$ is positive definite on $(K, S)$, if $f\left(X_{1}, \ldots, X_{m}\right)$ is positive definite on $K$ for every ordering of $(K, S) . f\left(X_{1}, \ldots, X_{m}\right)$ is said to be strongly positive definite on $(K, S)$, if $f\left(X_{1}, \ldots, X_{m}\right)$ is positive definite on every real closure of $(K, S)$. And $f\left(X_{1}, \ldots, X_{m}\right)$ is said to be totally positive definite on $(K, S)$, if $f\left(X_{1}, \ldots, X_{m}\right)$ is positive definite on every ordered extension of $(K, S)$. Obviously, we have the relations that totally positive definite $\Rightarrow$ strongly positive definite $\Rightarrow$ positive definite. Using Lang's Homomorphism Theorem or the model-completeness for real closed fields, it is easy to prove the following

PROPOSITION 3. If $(K, S)$ is a preordered field and

$$
f\left(X_{1}, \ldots, X_{m}\right) \in K\left(X_{1}, \ldots, X_{m}\right),
$$

then the following statements are equivalent:

(1) $f\left(X_{1}, \ldots, X_{m}\right)$ is strongly positive definite on $(K, S)$.

(2) $f\left(X_{1}, \ldots, X_{m}\right)$ is totally positive definite on $(K, S)$.

(3) $f\left(X_{1}, \ldots, X_{m}\right)$ can be represented in the form

$$
f\left(X_{1}, \ldots, X_{m}\right)=\sum_{i=1}^{n} s_{i} h_{i}^{2}\left(X_{1}, \ldots, X_{m}\right)
$$

where $s_{i} \in S, h_{i}\left(X_{1}, \ldots, X_{m}\right) \in K\left(X_{1}, \ldots, X_{m}\right)$ for $i=1, \ldots, n$.

Now we generalize the weak Hilbert property to preordered fields. We shall say that the preordered field $(K, S)$ has the weak Hilbert property, if every positive definite rational function is also strongly positive definite on $(K, S)$. By Proposition 3 , for a preordered field $(K, S)$ with the weak Hilbert property we have the relations that positive definite $\Leftrightarrow$ strongly positive definite $\Leftrightarrow$ totally positive definite, and every positive definite rational function can be represented in such form as in Proposition 3.

Finally, we give the following definitions.

DEFINITION 1. A finite extension $K^{*}$ of $K$ is said to be a finite real extension of $(K, S)$, if $K^{*}$ is formally real with respect to $S$; that is, -1 can not be expressed in the form $\sum_{i=1}^{n} s_{i} b_{i}^{2}$, where $s_{i} \in S, b_{i} \in K^{*}$ for $i=1, \ldots, n$.

By Definition 1, a finite real extension of $(K, S)$ has at least one ordering such that it contains $S$.

DEFINITION 2. A preordered field $(K, S)$ is said to be locally dense, if, for every finite real extension $K^{*}$ of $(K, S), \alpha, \beta \in K^{*}$ and $\alpha \neq \beta$, there exists always some $a \in K$ such that $a \in(\alpha, \beta)_{P^{*}}$ for some ordering $P^{*}$ of $K^{*}$ with $P^{*} \supseteq S$, where $(\alpha, \beta)_{P^{*}}$ the open interval with the endpoints $\alpha, \beta$ with respect to the ordering $P^{*}$.

REMARK. By the notation $(\alpha, \beta)_{P^{*}}$ we do not mean $\alpha<_{P^{*}} \beta$. 
2. Some lemmas. For the preliminary, in this section we give some lemmas. Here $K$ is always a formally real field, and $A$ is its algebraic closure. Without loss of generality, we may suppose that any algebraic extension of $K$ is always contained in $A$. Then, for an arbitrary real closure $(\bar{K}, \bar{P})$ of $K$, every element $\alpha$ in $A$ can be written as $\alpha=a+b \sqrt{-1}$, where $a, b \in \bar{K}$. In this case, we denote $\operatorname{Im}_{\bar{K}}(\alpha)=b$, and we may obtain the absolute value $|\alpha|_{\bar{P}}$ of $\alpha$ with respect to the ordering $\bar{P}$ by defining $|\alpha|_{\bar{P}}=\sqrt{a^{2}+b^{2}} \in \bar{P}$. It is easy to prove that the absolute value $|\cdot|_{\bar{P}}$ has the same properties as the usual absolute value of complex numbers.

LEMMA 1. If $f(X)$ is a polynomial of positive degree with coefficients in $K$, then there exist $\varepsilon, M \in \sum K^{2}$ such that for every real closure $(\bar{K}, \bar{P})$ of $K$, the following inequalities always hold: $\varepsilon<_{\bar{P}}|\alpha|_{\bar{P}}<_{\bar{P}} M$ where $\alpha$ is an arbitrary nonzero root of $f(X)$ in $A$.

PROOF. Let $f(X)=a_{n} X^{n}+a_{n-1} X^{n-1}+\cdots+a_{0}, a_{n} \neq 0$. Then, for every real closure $(\bar{K}, \bar{P})$ of $K$ and any $\operatorname{root} \alpha$ of $f(X)$ in $A$,

$$
|\alpha|_{\bar{P}}<_{\bar{P}} 1+\left|\frac{a_{n-1}}{a_{n}}\right|_{\bar{P}}+\cdots+\left|\frac{a_{0}}{a_{n}}\right|_{\bar{P}}<_{\bar{P}} n+1+\left(\frac{a_{n-1}}{a_{n}}\right)^{2}+\cdots+\left(\frac{a_{0}}{a_{n}}\right)^{2} .
$$

Hence let $M=n+1+\left(a_{n-1} / a_{n}\right)^{2}+\cdots+\left(a_{0} / a_{n}\right)^{2}$. When $\alpha \neq 0$, we have $a_{0}(1 / \alpha)^{n}+a_{1}(1 / \alpha)^{n-1}+\cdots+a_{n}=0$. Similarly, there exists some $\delta \in \sum K^{2}$ such that $|1 / \alpha|_{\bar{P}}<_{\bar{P}} \delta$. Hence let $\varepsilon=1 / \delta$.

LEMMA 2. If $f(X)$ is a polynomial of positive degree with coefficients in $K$, then there exists some $\varepsilon \in \sum K^{2}$ such that for evey real closure $(\bar{K}, \bar{P})$ of $K$, the inequality $\left|\operatorname{Im}_{\bar{K}}(\alpha)\right|_{\bar{P}}>_{\bar{P}} \varepsilon$ always holds where $\alpha$ is an arbitrary root of $f(X)$ in $A$, but not in $\bar{K}$.

ProOF. Putting $f(U+V \sqrt{-1})=f_{1}(U, V)+\sqrt{-1} f_{2}(U, V)$, where $U, V$ are indeterminates over $K, f_{i}(U, V) \in K[U, V]$ for $i=1,2$. We may assert that the two polynomials $f_{1}(U, V)$ and $f_{2}(U, V)$ have no nonconstant common factor in $K[U, V]$. Indeed, if $d(U, V)$ is a nonconstant factor of $f_{1}(U, V)$ and $f_{2}(U, V)$, then

$$
f(U+V \sqrt{-1})=d(U, V) \cdot\left[q_{1}(U, V)+\sqrt{-1} q_{2}(U, V)\right],
$$

where $q_{i}(U, V)=f_{i}(U, V) / d(U, V), i=1,2$. Let $a_{1}, \ldots, a_{m}$ be all the roots of $f(X)$, then

$$
f(U+V \sqrt{-1})=\left(U+V \sqrt{-1}-a_{1}\right) \cdots\left(U+V \sqrt{-1}-a_{m}\right) .
$$

Thus

$$
d(U, V)=b\left(U+V \sqrt{-1}-a_{i_{1}}\right) \cdots\left(U+V \sqrt{-1}-a_{i_{k}}\right),
$$

because $A[U, V]$ is a U.F.D. Let $\bar{K}$ be an arbitrary real closure of $K$, then $A[U, V]=$ $\bar{K}[U, V][\sqrt{-1}]$. Let $\tau$ be the $\bar{K}[U, V]$-automorphism of $A[U, V]$ such that $\tau(\sqrt{-1})=$ $-\sqrt{-1}$. Then

$$
d(U, V)=\tau(d(U, V))=\tau(b)\left(U-V \sqrt{-1}-\tau\left(a_{i_{1}}\right)\right) \cdots\left(U-V \sqrt{-1}-\tau\left(a_{i_{k}}\right)\right) .
$$

This contradicts the uniqueness of factorization of $d(U, V)$ in $A[U, V]$. Hence the resultant $R_{V}\left(f_{1}, f_{2}\right)$ of $f_{1}$ and $f_{2}$ relative to $U$ is a polynomial of positive degree in the indeterminate $V$. By Lemma 1, there exists some $\varepsilon \in \sum K^{2}$ such that for every real closure $(\bar{K}, \bar{P})$ of $K$, we have the inequality $|\beta|_{\bar{P}}>_{\bar{P}} \varepsilon$, where $\beta$ is an 
arbitrary nonzero root of $R_{V}\left(f_{1}, f_{2}\right)$. Obviously, for every root $\alpha$ of $f(X)$ which is not in $\bar{K}, \operatorname{Im}_{\bar{K}}(\alpha)$ is a nonzero root of $R_{V}\left(f_{1}, f_{2}\right)$. Therefore, we have the inequality $\left|\operatorname{Im}_{\bar{K}}(\alpha)\right|_{\bar{P}}>_{\bar{P}} \varepsilon$. This completes the proof.

LEMMA 3. If $f\left(X_{1}, \ldots, X_{m}\right) \in K\left[X_{1}, \ldots, X_{m}\right], a_{1}, \ldots, a_{m} \in A$, and $f\left(a_{1}, \ldots, a_{m}\right) \neq 0$, then there exists some $\delta \in \sum K^{2}$ such that for every real closure $(\bar{K}, \bar{P})$ of $K$ containing $a_{1}, \ldots, a_{m}$, whenever $\left|a_{i}-y_{i}\right|_{\bar{P}}<_{\bar{P}} \delta, y_{i} \in \bar{K}, i=1, \ldots, m$, the following inequality holds:

$$
f\left(y_{1}, \ldots, y_{m}\right) \cdot f\left(a_{1}, \ldots, a_{m}\right)>_{\bar{P}} 0 .
$$

PROOF. For every real closure $(\bar{K}, \bar{P})$ containing $a_{1}, \ldots, a_{m}$, with Taylor expansion we have the estimate

$$
\begin{aligned}
f(\bar{a}) \cdot f(\bar{a}+\bar{x})=f\left(a_{1}, \ldots, a_{m}\right) \cdot f\left(a_{1}+x_{1}, \ldots, a_{m}+x_{m}\right) \\
=f(\bar{a}) \cdot\left(f(\bar{a})+\sum_{i_{1}, \ldots, i_{m}} f_{i_{1}, \ldots, i_{m}}(\bar{a}) x_{1}^{i_{1}} \cdots x_{m}^{i_{m}}\right) \\
=f^{2}(\bar{a})+\sum_{i_{1}, \ldots, i_{m}} f(\bar{a}) \cdot f_{i_{1}, \ldots, i_{m}}(\bar{a}) x_{1}^{i_{1}} \cdots x_{m}^{i_{m}} \\
\quad>_{\bar{P}} f^{2}(\bar{a})-\sum_{i_{1}, \ldots, i_{m}}\left(1+f^{2}(\bar{a}) f_{i_{1}, \ldots, i_{m}}^{2}(\bar{a})\right)\left|x_{1}^{i_{1}}\right| \cdots\left|x_{m}^{i_{m}}\right| .
\end{aligned}
$$

Then, whenever $\left|x_{i}\right|_{\bar{P}}<_{\bar{P}} 1, i=1, \ldots, m$,

$$
f(\bar{a}) \cdot f(\bar{a}+\bar{x})>_{\bar{P}} f^{2}(\bar{a})-\sum_{i_{1}, \ldots, i_{m}}\left(1+f^{2}(\bar{a}) f_{i_{1}, \ldots, i_{m}}^{2}(\bar{a})\right)\left(\left|x_{1}\right|+\cdots+\left|x_{m}\right|\right)
$$

Putting

$$
Q=f^{2}(\bar{a}) / f^{2}(\bar{a})+m \cdot \sum_{i_{1}, \ldots, i_{m}}\left(1+f^{2}(\bar{a}) f_{i_{1}, \ldots, i_{m}}^{2}(\bar{a})\right),
$$

we have that $Q<_{\bar{P}} 1$, and whenever $\left|x_{i}\right|_{\bar{P}}<_{\bar{P}} Q, i=1, \ldots, m, f(\bar{a}) \cdot f(\bar{a}+\bar{x})>_{\bar{P}} 0$.

By Lemma 1, there exists some $\delta \in \sum K^{2}$ such that $|Q|_{\bar{P}}>_{\bar{P}} \delta$. Therefore $\delta$ is required.

3. Main results. Using the above lemmas, we can show the following

THEOREM 1. If $(K, S)$ is a preordered field, then the following statements are equivalent:

(1) $(K, S)$ is locally dense.

(2) For every finite real extension $K^{*}, a^{*} \in K^{*}, \varepsilon \in S$, there exists always some $a \in K$ such that $\left|a^{*}-a\right|<_{P^{*}} \varepsilon$, where $P^{*}$ is some ordering of $K^{*}$ with $P^{*} \supseteq S$.

(3) For every finite real extension $K\left(\theta^{*}\right), \varepsilon \in S$, there exist always some $\theta \in K$ such that $\left|\theta^{*}-\theta\right|<_{P^{*}} \varepsilon$, where $P^{*}$ is some ordering of $K\left(\theta^{*}\right)$ with $P^{*} \supseteq S$.

(4) For every finite real extension $K^{*}, a_{1}^{*}, \ldots, a_{m}^{*} \in K^{*}, \varepsilon \in S$, there exist always $a_{1}, \ldots, a_{m} \in K$ such that $\left|a_{i}^{*}-a_{i}\right|<_{P^{*}} \varepsilon, i=1, \ldots, m$, where $P^{*}$ is some ordering of $K^{*}$ with $P^{*} \supseteq S$.

(5) For every finite real extension $K^{*}, a_{1}^{*}, \ldots, a_{m}^{*} ; b_{1}^{*}, \ldots, b_{m}^{*} \in K^{*}$, and $a_{i}^{*} \neq b_{i}^{*}$ for $i=1, \ldots, m$, there exist always $c_{1}, \ldots, c_{m} \in K$ such that $c_{i} \in\left(a_{i}^{*}, b_{i}^{*}\right)_{P^{*}}$, $i=1, \ldots, m$, where $P^{*}$ is some ordering of $K^{*}$ with $P^{*} \supseteq S$. 
ProOF. $\quad(1) \Rightarrow(2)$ : By local density, there exists some $a$ in $K$ such that $a \in\left(a^{*}-\varepsilon, a^{*}+\varepsilon\right)_{P^{*}}$, where $P^{*}$ is some ordering of $K^{*}$ with $P^{*} \supseteq S$. Hence $\left|a^{*}-a\right|$ $<P^{*} \varepsilon$.

$(2) \Rightarrow(3)$. Obvious.

$(3) \Rightarrow(4)$. By the familiar Primitive Element Theorem, $K^{*}=K\left(\theta^{*}\right)$. Then $a_{i}^{*}=h_{i}\left(\theta^{*}\right)$ where $h_{i}(X) \in K[X], i=1, \ldots, m$. Considering the polynomial $\phi(X, Y)=\sum_{i=1}^{m}\left(h_{i}(X)-h_{i}(Y)\right)^{2}-\varepsilon^{2}$, by Lemma 3 there exists $\delta \in \sum K^{2}$ such that for every real closure $(\bar{K}, \bar{P})$ of $K$ containing $\theta^{*}$, whenever $\left|x-\theta^{*}\right|<_{\bar{P}} \delta$ and $\left|y-\theta^{*}\right|<_{\bar{P}} \delta, \phi(x, y) \cdot \phi\left(\theta^{*}, \theta^{*}\right)>_{\bar{P}} 0$, i.e. $\phi(x, y)<_{\bar{P}} 0$. By (3), there exists some $\theta \in K$ such that $\left|\theta^{*}-\theta\right|<P^{*} \delta$ where $P^{*}$ is some ordering of $K^{*}$ with $P^{*} \supseteq S$. Thereby $\phi\left(\theta^{*}, \theta\right)<P^{*} 0$, and

$$
\sum_{i=1}^{m}\left(a_{i}^{*}-h_{i}(\theta)\right)^{2}-\varepsilon^{2}=\sum_{i=1}^{m}\left(h_{i}\left(\theta^{*}\right)-h_{i}(\theta)\right)^{2}-\varepsilon^{2}=\phi\left(\theta^{*}, \theta\right)<P^{*} 0,
$$

further we have $\left|a_{i}^{*}-h_{i}(\theta)\right|<_{P^{*}} \varepsilon$. Therefore let $a_{i}=h_{i}(\theta), i=1, \ldots, m$.

$(4) \Rightarrow(5)$. By Lemma 1 , there exists $\varepsilon_{i} \in \sum K^{2}$ such that $\left|\left(a_{i}^{*}-b_{i}^{*}\right) / 2\right|>_{\bar{P}} \varepsilon_{i}$ for every real closure $(\bar{K}, \bar{P})$ of $K$. Putting $\varepsilon=\prod_{i=1}^{m}\left(\varepsilon_{i} / 1+\varepsilon_{i}\right)$, then $\varepsilon \in \sum K^{2} \subseteq S$. By (4), there exists $c_{i} \in K$ such that $\left|\left(a_{i}^{*}+b_{i}^{*}\right) / 2-c_{i}\right|<_{P^{*}} \varepsilon, i=1, \ldots, m$, where $P^{*}$ is some ordering of $K^{*}$ with $P^{*} \supseteq S$. Hence

$$
\left|\left(a_{i}^{*}+b_{i}^{*}\right) / 2-c_{i}\right|<_{P^{*}} \varepsilon<_{P^{*}} \varepsilon_{i}<_{P^{*}}\left|\left(a_{i}^{*}-b_{i}^{*}\right) / 2\right|,
$$

and $c_{i} \in\left(a_{i}^{*}, b_{i}^{*}\right)_{P^{*}}, i=1, \ldots, m$.

$(5) \Rightarrow(1)$. Obvious.

To characterize the weak Hilbert property by means of properties of polynomials in one variable, we give again a name. We will say that a preordered field $(K, S)$ has the property ${ }^{*}$, if, for every polynomial $f(X)$ in one variable $X$ which has a root in a real closure of $(K, S)$ and every nonzero element $z$ in $K$, there exists always some $w \in K$ such that $f^{2}(w)<_{Q} z^{2}$ for some ordering $Q$ of $(K, S)$. The property ${ }^{*}$ is similar to the implication of sentences of Theorem 5 in reference [1], and we have the following

THEOREM 2. The property * can be expressed by first order sentences in the language of preordered fields.

PROOF. Our proof follows Prestel's method of axiomatizing the class of prcfields [4]. For every natural number $n$, we consider the general polynomial $f_{n}(X)=$ $t_{n} X^{n}+t_{n-1} X^{n-1}+\cdots+t_{0}$, where $t_{0}, \ldots, t_{n}$ are parameters.

Obviously, a preordered field $(K, S)$ has the property ${ }^{*}$ if and only if for every natural number $n$ we have

$\left(H_{n}\right)$ For $t_{0}, \ldots, t_{n}, z \in K$, if for some $P \in X_{K}(S)$ there is $x \in(\bar{K}, \bar{P})$ with $f_{n}(x)=0$, and $z \neq 0$, then there exists some $w \in K$ such that $-f_{n}^{2}(w)+z^{2} \in Q$ for some $Q \in X_{K}(S)$.

We first show that the clause "for some $P \in X_{K}(S)$ there is $x \in(\bar{K}, \bar{P})$ with $f_{n}(x)=0$ " can be expressed in the language of preordered fields. By elimination of quantifiers for real closed fields we can replace "there is ..." by a quantifier free formula involving $P$. If we take this formula in disjunctive normal form, then it suffices to express in the language of preordered fields some clause of the type

"for some $P \in X_{K}(S): e(\mathbf{t})=0 \wedge q_{1}(\mathbf{t}) \in P \wedge \cdots \wedge q_{r}(\mathbf{t}) \in P$ " 
where $e, q_{1}, \ldots, q_{r}$ are polynomials with integral coefficients in the indeterminates $t$ 's. In fact, the clause is equivalent to the formula

$$
\left(\forall s_{i} \in S\right)\left(-1 \neq \sum_{i=1}^{2^{r}} b_{i} s_{i}\right)
$$

where the $b_{i}$ 's are $2^{r}$ distinct elements of the form $q_{1}^{k_{1}}(\mathbf{t}) \cdots q_{r}^{k_{r}}(\mathbf{t})$ with $k_{j}=0$ or 1 .

Similarly, the clause " $-f^{2}(w)+z^{2} \in Q$ for some $Q \in X_{K}(S)$ " can be also expressed in the language of preordered fields. This completes the proof.

Now, we can show the following main result:

THEOREM 3. If $(K, S)$ is a preordered field, then the following statements are equivalent:

(1) $(K, S)$ has the weak Hilbert property.

(2) Every positive definite polynomial in one variable is also strongly positive definite.

(3) $(K, S)$ has the property* .

(4) $(K, S)$ is locally dense.

ProOF. $(1) \Rightarrow(2)$. Obvious.

$(2) \Rightarrow(3)$. Let $f(X) \in K[X]$ have a $\operatorname{root} \bar{\theta}$ in some real closure $(\bar{K}, \bar{P})$ of $(K, S)$, and $0 \neq z \in K$. Then the polynomial $\phi(X)=f^{2}(X)-z^{2}$ is not strongly positive definite, because $\phi(\bar{\theta})=-z^{2}<_{\bar{P}} 0$. By $(2), \phi(X)$ is not positive definite on $(K, S)$, i.e. there exists $w$ in $K$ such that for some ordering $Q$ of $(K, S), f^{2}(w)-z^{2}<_{Q} 0$. Hence $(K, S)$ has the property ${ }^{*}$.

$(3) \Rightarrow(4)$. Let $K\left(\theta^{*}\right)$ be a finite real extension of $(K, S)$ and $\varepsilon \in S$. We will show that there exists some $\theta \in K$ such that $\left|\theta^{*}-\theta\right|<_{P^{*}} \varepsilon$, where $P^{*}$ is some ordering of $K\left(\theta^{*}\right)$ with $P^{*} \supseteq S$. Let $f(X)$ be the minimum polynomial of $\theta^{*}$ over $K$, and let $\varepsilon_{1} \in \sum K^{2}$ be as in Lemma 2 for the polynomial $f(X)$. Since $K\left(\theta^{*}\right)$ is a real extension of $(K, S)$, it has an ordering $P_{0}$ such that $P_{0} \supseteq S$. If $\left(\overline{K\left(\theta^{*}\right)}, \bar{P}_{0}\right)$ is the closure of the ordered field $\left(K\left(\theta^{*}\right), P_{0}\right)$, then $\left(\overline{K\left(\theta^{*}\right)}, \bar{P}_{0}\right)$ is also a real closure of $(K, S)$, and $f(X)$ has the root $\theta^{*}$ in $\overline{K\left(\theta^{*}\right)}$. By the property ${ }^{*}$, there exists some $\theta \in K$ such that for some ordering $P$ of $(K, S), f^{2}(\theta)<_{P} \Delta^{2 d}$, where $d$ is the degree of $f(X)$, and $\Delta=\varepsilon /(1+\varepsilon) \cdot \varepsilon_{1} /\left(1+\varepsilon_{1}\right)$. Hence we have $|f(\theta)|<_{P} \Delta^{d}$.

Let $(\bar{K}, \bar{P})$ be the real closure of the ordered field $(K, \dot{P})$, and $f(X)$ is factorized in the form

$$
f(X)=\left(X-\bar{\theta}_{1}\right) \cdots\left(X-\bar{\theta}_{s}\right) \cdot\left[\left(X+\bar{a}_{1}\right)^{2}+\bar{b}_{1}^{2}\right] \cdots\left[\left(X+\bar{a}_{t}\right)^{2}+\bar{b}_{t}^{2}\right]
$$

where $\bar{\theta}_{i}, \bar{a}_{j}, \bar{b}_{j} \in \bar{K}$, and $\bar{b}_{j} \neq 0, i=1, \ldots, s, j=1, \ldots, t, d=s+2 t$.

Hence, by $|f(\theta)|<_{P} \Delta^{d}$, we have

$$
\left|\theta-\bar{\theta}_{1}\right| \cdots\left|\theta-\bar{\theta}_{s}\right| \cdot\left|\left(\theta+\bar{a}_{1}\right)^{2}+\bar{b}_{1}^{2}\right| \cdots\left|\left(\theta+\bar{a}_{t}\right)^{2}+\bar{b}_{t}^{2}\right|<_{\bar{P}} \Delta^{d} .
$$

By Lemma 2, we have

$$
\left|\left(\theta+\bar{a}_{1}\right)^{2}+\bar{b}_{1}^{2}\right| \cdots\left|\left(\theta+\bar{a}_{t}\right)^{2}+\bar{b}_{t}^{2}\right| \geq_{\bar{P}} \bar{b}_{1}^{2} \cdots \bar{b}_{t}^{2}>_{\bar{P}} \varepsilon_{1}^{2} \cdots \varepsilon_{1}^{2}=\varepsilon_{1}^{2 t}>_{\bar{P}} \Delta^{2 t} .
$$

Thereby $d \neq 2 t$; i.e. $s \neq 0$. Furthermore $\left|\theta-\bar{\theta}_{1}\right| \cdots\left|\theta-\bar{\theta}_{s}\right|<_{\bar{P}} \Delta^{s}$. Therefore, for some $v, 1 \leq v \leq s,\left|\theta-\bar{\theta}_{v}\right|<_{\bar{P}} \Delta<_{\bar{P}} \varepsilon$. 
Let $\tau$ be the $K$-injection of $K\left(\theta^{*}\right)$ to $\bar{K}$ such that $\tau\left(\theta^{*}\right)=\bar{\theta}_{v}$. Then we may define an ordering $P^{*}$ of $K\left(\theta^{*}\right)$ as follows:

$$
\text { for } a^{*} \in K\left(\theta^{*}\right), \quad a^{*} \in P^{*} \quad \text { iff } \quad \tau\left(a^{*}\right) \in \bar{P} .
$$

Obviously, $P^{*} \supseteq S$, and $\left|\theta^{*}-\theta\right|<P^{*} \varepsilon$. By Theorem $1,(K, S)$ is locally dense.

$(4) \Rightarrow(1)$. It is sufficient to prove that every polynomial $f\left(X_{1}, \ldots, X_{m}\right)$, which is not strongly positive definite on $(K, S)$, is not positive definite on $(K, S)$. Since $f\left(X_{1}, \ldots, X_{m}\right)$ is not strongly positive definite, there exists $\bar{a}_{1}, \ldots, \bar{a}_{m}$ in some real closure $(\bar{K}, \bar{P})$ of $(K, S)$ such that $f\left(\bar{a}_{1}, \ldots, \bar{a}_{m}\right)<_{\bar{P}} 0$. Putting

$$
K^{*}=K\left(\bar{a}_{1}, \ldots, \bar{a}_{m}, \sqrt{-f\left(\bar{a}_{1}, \ldots, \bar{a}_{m}\right)}\right) \subseteq \bar{K},
$$

then $K^{*}$ is a finite real extension of $(K, S)$. For the polynomial $f\left(X_{1}, \ldots, X_{m}\right)$ and $\bar{a}_{1}, \ldots, \bar{a}_{m}$, there exists $\delta \in \sum K^{2}$ satisfying the condition in Lemma 3 . Since $(K, S)$ is locally dense, by Theorem $1, K^{*}$ has an ordering $P^{*} \supseteq S$, and there exist $a_{1}, \ldots, a_{m} \in K$ such that $\left|\bar{a}_{i}-a_{i}\right|<_{P^{*}} \delta, i=1, \ldots, m$. Then $f\left(\bar{a}_{1}, \ldots, \bar{a}_{m}\right)=$ $-\left(\sqrt{-f\left(\bar{a}_{1}, \ldots, \bar{a}_{m}\right)}\right)^{2}<_{P^{*}} 0$. Hence, by Lemma $3, f\left(a_{1}, \ldots, a_{m}\right)<_{P^{*}} 0$; that is, $f\left(X_{1}, \ldots, X_{m}\right)$ is not positive definite on $(K, S)$.

To illustrate an application of the above results, we show the following

THEOREM 4. If $(K, S)$ is a preordered field with the weak Hilbert property, and $P \in X_{K}(S)$ is isolated for Harrison's topology, then the ordered field $(K, P)$ is dense in its real closure.

ProOF. Let $(\bar{K}, \bar{P})$ be the real closure of the ordered field $(K, P)$. It suffices to show that for $\bar{a} \in \bar{K}, \varepsilon \in P$ there exists some $a \in K$ such that $|\bar{a}-a|<_{\bar{P}} \varepsilon$. Let $\bar{a}_{1}=\bar{a}, \bar{a}_{2}, \ldots, \bar{a}_{v}$ be all of the conjugate elements of $\bar{a}$ in $\bar{K}$ over $K$. Since $P$ is isolated, there is a basic open set $H\left(b_{1}, \ldots, b_{n}\right)$ such that $H\left(b_{1}, \ldots, b_{n}\right)=\{P\}$. Putting $K^{*}=K\left(\bar{a}_{1}, \ldots, \bar{a}_{v}, \sqrt{b_{1}}, \ldots, \sqrt{b_{n}}\right)$, then $K^{*} \subseteq \bar{K}$ and $K^{*}$ is a finite real extension of $(K, S)$. By Theorem $3,(K, S)$ is locally dense. Hence, by Theorem 1 , there exist $a_{1}, \ldots, a_{v} \in K$ such that $\left|\bar{a}_{i}-a_{i}\right|<_{P^{*}}(\varepsilon / 1+\varepsilon)^{2}$ for $i=1, \ldots, v$, where $P^{*}$ is some ordering of $K^{*}$ with $P^{*} \supseteq S$. Notice that $b_{j}=\left(\sqrt{b_{j}}\right)^{2} \in P^{*} \cap K$ for $j=1, \ldots, n$. Thereby $P^{*} \cap K \in H\left(b_{1}, \ldots, b_{n}\right)=\{P\}$, i.e. $P^{*} \cap K=P$, and $\left(K^{*}, P^{*}\right)$ is an ordered algebraic extension of the ordered field $(K, P)$. So there is an orderpreserving $K$-injection $\tau$ of $K^{*}$ to $\bar{K}$, and there is some $\bar{a}_{k}, 1 \leq k \leq v$, such that $\tau\left(\bar{a}_{k}\right)=\bar{a}$. By $\left|\bar{a}_{k}-a_{k}\right|<_{P^{*}}(\varepsilon / 1+\varepsilon)^{2}$, we have $\left|\tau\left(\bar{a}_{k}\right)-\tau\left(a_{k}\right)\right|<_{\bar{P}} \tau\left((\varepsilon / 1+\varepsilon)^{2}\right)$; that is, $\left|\bar{a}-a_{k}\right|<_{\bar{P}}(\varepsilon / 1+\varepsilon)^{2}<_{\bar{P}} \varepsilon$. Therefore $(K, P)$ is dense in $(\bar{K}, \bar{P})$.

Finally, by Theorem 4 , we easily obtain the following result:

COROLLARY. Suppose that a preordered field $(K, S)$ has only a finite number of orderings. Then $(K, S)$ has the weak Hilbert property if and only if $K$ is dense in every real closure of $(K, S)$.

ProOF. " $\Leftarrow$ ": Obvious.

" $\Rightarrow$ ". Since $X_{K}(S)$ is a Hausdorff topological space of finitely many points, every point of $X_{K}(S)$ is isolated. By Theorem 4, for every $P \in X_{K}(S)$ the ordered field $(K, P)$ is dense in its real closure. This completes the proof of the "only if" part.

REMARK. When $S$ is exactly an ordering of $K$ or $S=\sum K^{2}$, by the above Corollary, we immediately obtain Proposition 1 or Proposition 2 in the introduction, respectively. 
ACKNOWLEDGEMENT. The author is grateful to the referee for his helpful suggestions, especially those concerning Theorem 2 .

\section{REFERENCES}

1. K. McKenna, New facts about Hilbert's 17th problem, Lecture Notes in Math., vol. 498, Springer-Verlag, Berlin and New York, 1975, pp. 220-230.

2. A Prestel, Sums of squares over fields, Soc. Brasil Rio de Janeiro, 1979, pp. 33-44.

3. __ Lectures on formally real fields, IMPA Lecture Notes, no. 22, Soc. Brasil Rio de Janeiro, 1975.

4. __, Pseudo real closed fields, Lecture Notes in Math., vol. 872, Springer-Verlag, Berlin and New York, 1981, pp. 127-156.

5. T. Y. Lam, The theory of ordered fields, Lecture Notes in Pure and Appl. Math., vol. 55, Marcel Dekker, New York, 1980, pp. 1-152.

6. A. Robinson, On ordered fields and definite functions, Math. Ann. 130 (1955), 257-271.

7. N. Jacobson, Basic algebra. I, Freeman, San Francisco, Calif., 1974.

8. Zeng Guangxin, Positive definite functions over a formally real field with a core, Adv. in Math. (Chinese), 17 (1988), 285-289.

Department of Mathematics, Fuzhou Teacher's College, Jiangxi, The PeoPLE'S REPUBLIC OF CHINA 